\title{
Math-Net.Ru
}

Общероссийский математический портал

О. Г. Смолянов, А. Трумен, Формулы Фейнмана для решений уравнений Шредингера на компактных римановых многообразиях, Матем. заметки, 2000, том 68, выпуск $5,789-793$

DOI: https://doi.org/10.4213/mzm1000 
Использование Общероссийского математического портала Math-Net.Ru подразумевает, что вы прочитали и согласны с пользовательским соглашением http://www. mathnet.ru/rus/agreement

Параметры загрузки:

IP: 54.237 .206 .68

26 апреля 2023 г., 15:20:53

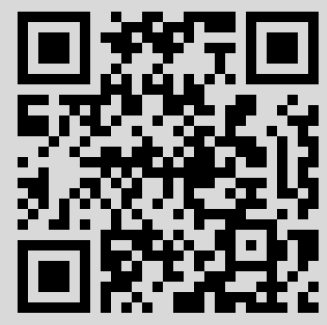




\section{ФОРМУЛЫ ФЕЙНМАНА ДЛЯ РЕШЕНИЙ УРАВНЕНИЙ ШРЁДИНГЕРА НА КОМПАКТНЫХ РИМАНОВЫХ МНОГООБРАЗИЯХ}

\section{О.Г. Смолянов, А. Трумен}

В заметке получены представления решений уравнений Шрёдингера на компактных римановых многообразиях с помощью формул типа Фейнмана и Фейнмана-Каца [1]-[3]; подобные представления существуют также для аналогичных стохастических уравнений. При этом в подынтегральных выражениях появляются дополнительные множители, зависящие от геометрии риманова многообразия (а если оно вложено в евклидово пространство, то и от характера вложения) [4]; они могут быть интерпретированы как соответствующие дополнительным потенциальным членам в уравнениях, что согласуется с физической парадигмой, согласно которой влияние геометрии риманова пространства может быть описано с помощью некоторого потенциала.

1. Меры на множестве траекторий в компактном римановом многообразии. Если $T$ и $G$ - топологические пространства, то через $C(T, G)$ обозначается множество всех непрерывных отображений из $T$ в $G$, наделенное компактно-открытой топологией; символ $C_{b}(T)$ обозначает пространство всех вещественных ограниченных функций на $T$, наделенное равномерной нормой. Всюду далее $G$ - это компактное риманово многообразие размерности $d>0$, изометрически вложенное в евклидово пространство $\mathbb{R}^{n}, \rho$ - риманово расстояние в $G$; для вского $t>0$ $E^{t}=C\left([0, t], \mathbb{R}^{n}\right), E_{G}^{t}=C([0, t], G)$, так что $E_{G}^{t} \subset E^{t}$ (это вложение индуцируется вложением $\left.G \subset \mathbb{R}^{n}\right)$. Пусть функции $p$ и $p_{R}$ на $\mathbb{R}^{n} \times G \times G$ определяются равенствами

$$
p\left(t, x_{1}, x_{2}\right)=(2 \pi t)^{-n / 2} e^{-\left\|x_{2}-x_{1}\right\|^{2} /(2 t)}
$$

Работа выполнена при финансовой поддержке Российского фонда фундаментальных исследований, грант № 99-01-01212. 
и

$$
p_{R}\left(t, x_{1}, x_{2}\right)=(2 \pi t)^{-n / 2} e^{-\rho^{2}\left(x_{1}, x_{2}\right) /(2 t)}
$$

пусть еще $p_{G}$ - это ядро интегрального оператора, решающего задачу Коши для уравнения теплопроводности на $G$. Для каждого $z \in \mathbb{R}^{n}$ символом $W^{z}$ обозначается стандартная мера Винера на $E^{t}$, сосредоточенная на функциях $\varphi \in E^{t}$ таких, что $\varphi(0)=z$, и символом $W_{G}^{z}$ - аналогичная мера Винера на $E_{G}^{t}$. Для всякого разбиения $\pi=\left\{t_{j}: 0=t_{0}<t_{1}<t_{2}<\cdots<t_{N}=t\right\}$ отрезка $[0, t]$ пусть $d(\pi)$ - это диаметр разбиения $\left(d(\pi)=\max \left|t_{j}-t_{j-1}\right|\right) ;$ для всякого $z \in G$ и $f \in C_{b}\left(E_{G}^{t}\right)$ пусть $f_{\pi}^{z}$ - функция на произведении $N$ копий $G$, определенная равенством $f_{\pi}^{z}\left(z_{1}, \ldots, z_{N}\right)=$ $f\left(\varphi_{\pi}^{\left.z, z_{1}, \ldots, z_{N}\right)}\right)$, где $\varphi_{\pi}^{z, z_{1}, \ldots, z_{N}} \in E_{G}^{t}, \varphi_{\pi}^{z, z_{1}, \ldots, z_{N}}\left(t_{0}\right)=z, \varphi_{\pi}^{z, z_{1}, \ldots, z_{N}}\left(t_{j}\right)=z_{j}(j>0)$ и для $s \in\left(t_{j}, t_{j+1}\right) \varphi_{\pi}^{z, z_{1}, \ldots, z_{N}}(s)=\gamma(s)$, где $\gamma \in C\left(\left[t_{j}, t_{j+1}\right], G\right),\left\|\gamma^{\prime}(\cdot)\right\|=$ const (норма в $\left.\mathbb{R}^{n}\right)$ и $\gamma\left(\left[t_{j}, t_{j+1}\right]\right)$ - (некоторая) минимальная геодезическая, соединяющая $z_{j}$ и $z_{j+1}$ (таким образом, $f_{\pi}^{z}$ определяется неоднозначно). Как показано в [4], существует такая вероятностная мера $\nu_{G}^{z}$ на $E_{G}^{t}$ (называемая поверхностной мерой, порожденной мерой $W^{z}$ ), что для всякой $f \in C_{b}\left(E_{G}^{t}\right)$

$$
\begin{gathered}
\int_{E_{G}^{t}} f(x) \nu_{G}^{z}(d x)=\lim _{d(\pi) \rightarrow 0} c_{N} \int_{G \times \cdots \times G} f_{N}^{z}\left(z_{1}, \ldots, z_{N}\right) p\left(t_{1}, z, z_{1}\right) \\
\times p\left(t_{2}-t_{1}, z-1, z_{2}\right) \cdots p\left(t_{N}-t_{N-1}, z_{N-1}, z_{N}\right) d z \ldots d z_{N}, \\
c_{N}^{-1}=\int_{G \times \cdots \times G} p\left(t_{1}, z, z_{1}\right) p\left(t_{2}-t_{1}, z_{1}, z_{2}\right) \cdots p\left(t_{N}-t_{N-1}, z_{N-1}, z_{N}\right) d z_{1} \ldots d z_{N}
\end{gathered}
$$

(предел не зависит от выбора $f_{\pi}^{z}$ ), где символом $\int_{G \times \cdots \times G} \ldots d z_{1} \ldots d z_{N}$ обозначают интегралы по мерам, являющимся произведениями мер на $G$, порождаемых формой объема на $G$; эта же форма объема используется при определении пространства $\mathscr{L}_{2}(G)$. Кроме того. согласно [4] существует вероятностная мера $\eta_{G}^{z}$ на $E_{G}^{t}$, определяемая аналогично мере $\nu_{G}^{z}$ с заменой $p$ на $p_{R}$. В [4] также показано, что

$$
\frac{d W_{G}^{z}}{d \eta_{G}^{z}}(h)=e^{\frac{1}{6} \int_{0}^{t} \operatorname{scal}(h(\tau)) d \tau}
$$

где для всякого $x \in G \operatorname{scal}(x)$ - скалярная кривизна в точке $x$ (в принятой здесь нормировке скалярной кривизны ее значение для единичной сферы $n$-мерного евклидова пространства равно $n(n-1))$, и что

$$
\frac{d W_{G}^{z}}{d \nu_{G}^{z}}=e^{\frac{1}{4} \int_{0}^{t} \operatorname{scal}(h(\tau))-\frac{1}{2}\left(c^{2}(h(\tau))\right) d \tau}
$$

где $c(x)$ - средняя кривизна многообразия $G$ в точке $x$, умноженная на его размерность.

2. Формулы Фейнмана-Каца для уравнений Шрёдингера на компактных римановых многообразиях. Всюду далее $\Delta$-это оператор Бельтрами-Лапласа в пространстве функций на $G$ и $V$ - некоторая функция на $G$.

Ниже рассматриваются уравнение теплопроводности

$$
\frac{\partial \varphi}{\partial t}=\frac{1}{2} \Delta \varphi+V \varphi
$$

и уравнение Шрёдингера

$$
\frac{\partial \psi}{\partial t}=i \frac{1}{2} \Delta \psi+i V \psi
$$

на $G$. При этом предполагается, что $\varphi$ и $\psi$ - функции на $[0, \infty)$, принимающие значения в некотором пространстве функций на $G$. 
ПРЕДЛОЖЕНИЕ 1. Если $V \in \mathscr{L}_{2}(G)$ u $\eta \in \mathscr{L}_{2}(G)$, то существует решение $\varphi$ задачи Коши для уравнения (1) такое, что

$$
\begin{aligned}
& \varphi(t)(x)=\int_{E_{G}^{t}} \eta(\xi(t)) \exp \left(\int_{0}^{t} V(\xi(\tau)) d \tau+\frac{1}{6} \int_{0}^{t} \operatorname{scal}(h(\tau)) d \tau\right) \eta_{G}^{x}(d \xi) \\
& =\int_{E_{G}^{t}} \eta(\xi(t)) \exp \left(\int_{0}^{t} V(\xi(\tau)) d \tau+\frac{1}{4} \int_{0}^{t}\left(\operatorname{scal}\left(h(\tau)-\frac{1}{2} c^{2}(h(\tau))\right)\right) d \tau\right) \nu_{G}^{x}(d \xi) .
\end{aligned}
$$

ДокАЗАТЕльство. Как первое, так и второе равенства могут быть доказаны с помощью теоремы Чернова и разложения в ряд Тейлора риманова тензора кривизны; при доказательстве первого равенства может быть также использована вместо разложения Тейлора формула для объемов "малых шаров" в римановом пространстве (ср. [4])

ЗАмечаниЕ 1. Из предложения 1 и каждого из приведенных выражений для производных $d W_{G}^{z} / d \eta_{G}^{z}$ и $d W_{G}^{z} / d \nu_{G}^{z}$ вытекает также (известное) представление решения $\varphi$ с помощью интеграла по мере $W_{G}^{z}$.

Теорема 1. Пусть $V$ и $\eta$ - это сужения на $G$ некоторых функций, определенных и аналитических на области в $\mathbb{C}^{n}$, содержащей $\mathbb{R}^{n} u \sqrt{i} \mathbb{R}^{n}$ (они обозначаются теми же символами). Тогда существует решение задачи Коши для уравнения (2) с начальными данными $(0, \eta)$ такое, что (ср. [5]-[7])

$$
\begin{aligned}
\int_{E_{G}^{t}} & \eta(\sqrt{i} \xi(t)) \exp \left(\int_{0}^{t} i V(\sqrt{i} \xi(\tau)) d \tau\right) W_{G}^{x}(d \xi) \\
= & \int_{E_{G}^{t}} \eta(\sqrt{i} \xi(t)) \exp \left(\int_{0}^{t} i V(\sqrt{i} \xi(\tau)) d \tau+\frac{1}{6} \int_{0}^{t} \operatorname{scal}(\xi(\tau)) d \tau\right) \eta_{G}^{x}(d \xi)
\end{aligned}
$$

Справедлив и аналог второго равенства из предолжения 1.

ДокАЗАТЕльСтво. Если $V_{1}(x)=V(\sqrt{i} x)$ и $\eta_{1}(x)=\eta(\sqrt{i} x)$, то в силу предложения 1 выражение справа определяет решение $\varphi$ задачи Коши для уравнения теплопроводности

$$
\frac{\partial \varphi}{\partial t}=\frac{1}{2} \Delta \varphi+V_{1} \varphi
$$

с начальными условиями $\left(0, \eta_{1}\right)$; но это значит, что функция $t \mapsto[x \mapsto \varphi(x / \sqrt{i})]$ является решением задачи Коши для уравнения $(2)$ с начальными условиями $\varphi(0)=\eta$. Следовательно, в силу формулы для производной Радона-Никодима $d W_{G}^{z} / d \eta_{G}^{z}$ и среднее выражение определяет решение той же задачи Коши. Другое доказательство можно получить, проверив с помощью формулы Ито, что среднее выражение в цепочке равенств определяет решение задачи Коши для уравнения (2) с начальньми данными $(0, \eta)$, и затем вновь воспользовавшись выражением для производной Радона-Никодима (справедлива и аналогичная формула, содержащая интегрирование по мере $\nu_{G}^{z}$ ).

3. Формула Фейнмана для уравнений Шрёдингера на компактных римановых многообразиях. Пусть $p_{\varphi}$ и $p_{\varphi}^{R}$ - функции на $(0, \infty) \times \mathbb{R}^{n} \times \mathbb{R}^{n}$, определяемые так:

$$
p_{\varphi}\left(t, x_{1}, x_{2}\right)=(2 \pi t)^{-d / 2} e^{i\left\|x_{2}-x_{1}\right\| /(2 t)} ; \quad p_{\varphi}^{R}\left(t, x_{1}, x_{2}\right)=(2 \pi t)^{-d / 2} e^{i p^{2}\left(x_{1}, x_{2}\right) /(2 t)} .
$$

Функция $f: E_{G}^{t} \rightarrow \mathbb{C}$ называется интегрируемой по поверхностной мере Фейнмана $\Phi_{G}^{z}$ или $\Phi_{G}^{z}$-интегрируемой, если существует (называемый поверхностньм интегралом Фейнмана и) обозначаемый символом $\int_{E_{G}^{t}} f(x) \Phi_{G}^{z}(d x)$ предел

$$
\begin{aligned}
& \lim _{d(\pi) \rightarrow 0} \alpha_{N} \int_{G \times \cdots \times G} f_{N}^{z}\left(z_{1}, \ldots, z_{N}\right) p_{\varphi}\left(t_{1}, z, z_{1}\right) \\
& \quad \times p_{\varphi}\left(t_{2}-t_{1}, z_{1}, z_{2}\right) \cdots p_{\varphi}\left(t_{N}-t_{N-1}, z_{N-1}, z_{N}\right) d z_{1} \ldots d z_{N} \\
& \left(\alpha_{N}\right)^{-1}=\int_{G \times \cdots \times G} p_{\varphi}\left(t_{1}, z, z_{1}\right) \\
& \quad \times p_{\varphi}\left(t_{2}-t_{1}, z_{1}, z_{2}\right) \cdots p_{\varphi}\left(t_{N}-t_{N-1}, z_{N-1}, z_{N}\right) d z_{1} \ldots d z_{N} .
\end{aligned}
$$




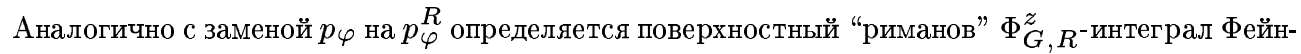
мана. Далее предполагается, что тензор римановой кривизны обладает аналитическим продолжением на $G+\sqrt{i} G(\subset \mathbb{C})$.

Теорема 2. В предположениях теоремы 1 существует решение $\varphi$ задачи Коши для (2) с начальными условиями $(0, \eta)$ такое, что

$$
\varphi(t)(x)=\int_{E_{G}^{t}} \eta(\xi(t)) \exp \left(\int_{0}^{t}\left(i V(\xi(\tau)) d \tau+\frac{1}{6} \operatorname{scal}\left(\frac{\xi(\tau)}{\sqrt{i}}\right)\right) d \tau\right) \Phi_{G, R}^{x}(d \xi) .
$$

ДокАЗАТЕльство. Интеграл Фейнмана в правой части последнего равенства по определению совпадает с пределом некоторой последовательности конечнократных интегралов; применяя к ним формулу замены переменной, можно получить последовательность интегралов, сходящуюся к интегралу в правой части цепочки равенств из теоремы 1 . Это означает, что исходная последовательность конечнократных интегралов также сходится, причем ее предел определяет решение задачи Коши для уравнения (2) с начальньми данньми $(0, \eta)$.

ЗАмеч Ание 2 . Справедлива и формула, аналогичная формуле (5), но содержащая $\Phi_{G}^{x}$-интеграл Фейнмана.

ЗАмечАниЕ 3 . При других предположениях о функциях $V$ и $\eta$ представление решения задачи Коши для (2) (с начальными условиями $(0, \eta))$ с помощью $\Phi_{G, R^{-}}^{x}$ или $\Phi_{G}^{x}$-интеграла Фейнмана можно также получить независимо от теоремы 1 с помощью формулы Чернова.

ЗАмечание 4. Определяемое естественным образом произведение $\left(d W_{G}^{z} / d \eta_{G}^{z}\right)(\sqrt{i} \cdot) \Phi_{G, R}^{z}$ можно назвать фейнмановской мерой на траекториях в $G$. В частности, функцию

$$
\xi \mapsto e^{\frac{i}{2} \int_{0}^{t}\left(\xi^{\prime}(\tau)\right)^{2} d \tau} e^{\frac{1}{6} \int_{0}^{t} \operatorname{scal}(\sqrt{i} \xi(\tau)) d \tau}
$$

можно считать обобщенной плотностью фейнмановской меры на $E_{G}^{t}$ (cp. [4]).

4. Интегралы по пространству траекторий в фазовом пространстве. Рассматривается задача Коши для уравнения (Шрёдингера)

$$
i \frac{\partial \varphi}{\partial t}=\widehat{\mathscr{H}} \varphi(t),
$$

где $\widehat{\mathscr{H}}$ - псевдодифференциальный оператор с символом $\mathscr{H}$, определенный на кокасательном расслоении $T^{*} G$ многообразия $G$. Предполагается, что вложение $G \subset \mathbb{R}^{n}$ обладает продолжением до изометрического вложения (в $\left.\mathbb{R}^{n}\right)$ расслоения $T^{*} G$.

Положим $T^{*} E_{G}^{t}=C\left([0, t], T^{*} G\right)$, и пусть символ $\int_{T^{*} E_{G}^{t}} \ldots \Phi_{T^{*} G}^{z}(d q d p)$ обозначает интеграл по секвенциальной (гамильтоновой) мере Фейнмана на $T^{*} E_{G}^{t}$, определение которой аналогично использованному в [1]. При подходящих ограничениях аналитического характера на символ $\mathscr{H}$ и функцию $\eta: G \rightarrow \mathbb{C}$ справедливо следующее предложение.

ПРЕДЛОЖЕнИЕ 2. Существует решение $\varphi$ задачи Коши для последнего уравнения $c$ начальным условием $(0, \eta)$ такое, что

$$
\begin{aligned}
\varphi(t)(z)= & \int_{E_{G}^{t}} \eta(\xi(t)) \exp \left(\int_{0}^{t} \mathscr{H}(q(\tau), p(\tau)) d \tau\right. \\
& \left.+\frac{1}{4} \int_{0}^{t}\left(\left(\operatorname{scal}\left(v_{1}(\tau), v_{2}(\tau)\right)-\frac{1}{2} c^{2}\left(v_{1}(\tau), v_{2}(\tau)\right)\right)\right) d \tau\right) \Phi_{T^{*} G}^{z}(d q d p),
\end{aligned}
$$

где $v_{1}(\tau)=\sqrt{i}\left(\int_{0}^{\tau} p(s) d s+q(\tau)\right), v_{2}(\tau)=\sqrt{i}\left(\int_{0}^{\tau} p(s) d s-q(\tau)\right)$, причем предполагается, что функиии $\mathrm{scal}($ ) и $\mathrm{c}($ ) обладают соответствующими аналитическими продолэениями.

После применения к конечнократным интегралам, аппроксимирующим дающий решение задачи Коши интеграл по поверхностной мере в $T^{*} E_{G}^{t}$ (определяемой аналогично мере $\nu_{G}^{t}$ ), замены переменной, аналогичной использованной в [6] (cp. [1]), получается последовательность интегралов, предел которой совпадает с интегралом Фейнмана; затем можно использовать теорему Чернова (cp. [4]). 


\section{СПИСОК ЦИТИРОВАННОЙ ЛИТЕРАТУРЫ}

1. Смолянов О. Г., Шавгулидзе Е. Т. Континуальные интегралы. М.: Изд-во МГУ, 1990. 2. Маслов В.П. Комплексные марковские цепи и континуаљный интеграл Фейнмана для нелинейных систем. M.: Наука, 1976. 3. Albeverio S., Hoegh-Krohn R. Mathematical Theory of Feynman Path Integrals // Lecture Notes in Math. V. 523. Berlin: Springer, 1976. 4. Вайцзеккер Х. фон, Смолянов О. Г., Виттих О. // Докл. РАН. 2000. Т. 371. №4. С. 442-447. 5. Albeverio S., Kolokol'tsov V. N., Smolyanov O. G. // C. R. Acad. Sci. Paris. 1996. V. 323. № 1. Р. 661-664. 6. Белавкин В. П., Смолянов О. Г. // Докл. РАН. 1998. Т. 360. № 5. C. 589-593. 7. Doss H. // Communications in Math. Phys. 1980. V. 73. P. 247-264.

Московский государственный университет им. М. В. Ломоносова 